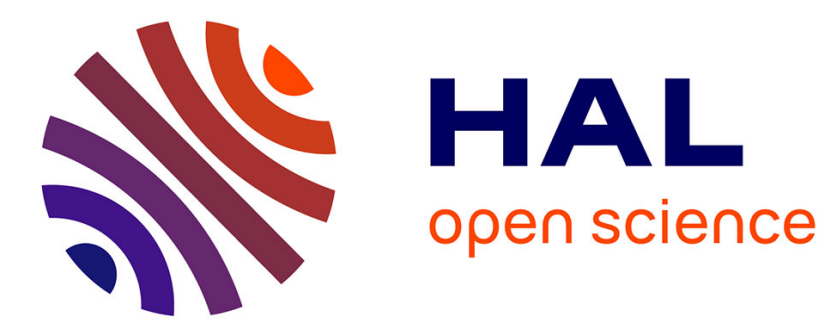

\title{
Hysteretic and intermittent regimes in the subcritical bifurcation of a quasi 1D system of interacting particles
} Tommy Dessup, Christophe Coste, Michel Saint-Jean

\section{To cite this version:}

Tommy Dessup, Christophe Coste, Michel Saint-Jean. Hysteretic and intermittent regimes in the subcritical bifurcation of a quasi 1D system of interacting particles. Physical Review E , 2016, 10.1103/PhysRevE.93.012105 . hal-01216045v2

\section{HAL Id: hal-01216045 \\ https://hal.science/hal-01216045v2}

Submitted on 29 Nov 2016

HAL is a multi-disciplinary open access archive for the deposit and dissemination of scientific research documents, whether they are published or not. The documents may come from teaching and research institutions in France or abroad, or from public or private research centers.
L'archive ouverte pluridisciplinaire HAL, est destinée au dépôt et à la diffusion de documents scientifiques de niveau recherche, publiés ou non, émanant des établissements d'enseignement et de recherche français ou étrangers, des laboratoires publics ou privés. 


\title{
Hysteretic and intermittent regimes in the subcritical bifurcation of a quasi-one-dimensional system of interacting particles
}

\author{
Tommy Dessup, Christophe Coste, and Michel Saint Jean \\ Laboratoire "Matière et Systèmes Complexes" (MSC), UMR 7057 CNRS, Université Paris 7 Diderot, 75205 Paris Cedex 13, France
}

(Received 30 September 2015; revised manuscript received 24 November 2015; published 7 January 2016)

\begin{abstract}
In this article, we study the effects of white Gaussian additive thermal noise on a subcritical pitchfork bifurcation. We consider a quasi-one-dimensional system of particles that are transversally confined, with shortrange (non-Coulombic) interactions and periodic boundary conditions in the longitudinal direction. In such systems, there is a structural transition from a linear order to a staggered row, called the zigzag transition. There is a finite range of transverse confinement stiffnesses for which the stable configuration at zero temperature is a localized zigzag pattern surrounded by aligned particles, which evidences the subcriticality of the bifurcation. We show that these configurations remain stable for a wide temperature range. At zero temperature, the transition between a straight line and such localized zigzag patterns is hysteretic. We have studied the influence of thermal noise on the hysteresis loop. Its description is more difficult than at $T=0 \mathrm{~K}$ since thermally activated jumps between the two configurations always occur and the system cannot stay forever in a unique metastable state. Two different regimes have to be considered according to the temperature value with respect to a critical temperature $T_{c}\left(\tau_{\mathrm{obs}}\right)$ that depends on the observation time $\tau_{\mathrm{obs}}$. An hysteresis loop is still observed at low temperature, with a width that decreases as the temperature increases toward $T_{c}\left(\tau_{\mathrm{obs}}\right)$. In contrast, for $T>T_{c}\left(\tau_{\mathrm{obs}}\right)$ the memory of the initial condition is lost by stochastic jumps between the configurations. The study of the mean residence times in each configuration gives a unique opportunity to precisely determine the barrier height that separates the two configurations, without knowing the complete energy landscape of this many-body system. We also show how to reconstruct the hysteresis loop that would exist at $T=0 \mathrm{~K}$ from high-temperature simulations.
\end{abstract}

DOI: 10.1103/PhysRevE.93.012105

\section{INTRODUCTION}

There has been much interest recently in physical systems constituted by interacting particles confined in narrow channels in quasi-one-dimensional geometry. Their typical size extends several orders of magnitude, from laser-cooled ions confined in Paul traps [1-14] and polar molecules [15], to optically confined paramagnetic colloidal particles [16], to plasma dusts in electrostatic traps [17-21], and to electrostatically interacting macroscopic beads [14,22-24]. The transverse confining potential is well described as harmonic (see [6] for an anharmonic potential), while the repulsive interactions may be described by the Coulomb potential [1-13], a dipolar interaction $[15,16]$, a Yukawa potential $U_{0} \exp \left(-r / \lambda_{0}\right) / r$ for screened electrostatic interaction [17-21], or an interaction potential $U_{0} K_{0}\left(r / \lambda_{0}\right)$ [14,22-24], where $K_{0}(\cdot)$ is the modified Bessel function of order 0 , and where $U_{0}$ and $\lambda_{0}$ are, respectively, the intensity and the range of interaction.

The behavior of these systems results from the interplay between the repulsive interparticle interactions and the potential that confines the particles in the transverse direction of the trap, which may be characterized by its stiffness $\beta$. When $\beta$ is large enough, all particles are aligned along the trap axis at equilibrium. As $\beta$ decreases below a critical stiffness $\beta_{Z Z}$, the equilibrium pattern undergoes a structural phase transition. The characteristics of the transition depend on the range of the interparticle interactions, on the boundary conditions in the longitudinal direction, and on the system size.

For an infinite interaction range $\left(\lambda_{0} \rightarrow \infty\right)$, typically for Coulombic interactions, a system always evolves from a straight line toward one of two regular symmetric staggered rows. This transition is therefore called the zigzag transition. Its relevant order parameter is the height $h$ defined as the distance between any particle and the longitudinal axis. A suitable control parameter is the dimensionless transverse stiffness $\epsilon=1-\beta / \beta_{Z Z}$ (see also [25]). The bifurcation diagram $h(\epsilon)$ corresponds to a supercritical pitchfork bifurcation: The zigzag phase spontaneously breaks the mirror symmetry, the zigzag phase is stable for $\epsilon \geqslant 0$, and its amplitude $h(\epsilon)$ is proportional to $\epsilon^{1 / 2}[1-4,13,14]$ [see Fig. 1(a)].

For longitudinally confined systems, and regardless of the interparticle interaction range, the same kind of transition can be observed. Because of the longitudinal boundary conditions, the two symmetric zigzag configurations are now inhomogeneous, with the highest distance $h_{m}$ always localized at the center of the finite cell, and a zigzag amplitude that symmetrically decreases toward both edges of the channel. In this case, the relevant order parameter is the maximum zigzag amplitude $h_{m}$. Its evolution with the dimensionless transverse stiffness $\epsilon$ evidences the supercritical nature of the bifurcation $[3,4,7,20,22,26]$.

In a finite periodic system with $N$ particles contained in a cell of length $L$, when the interparticle interaction is of finite range $\left(\lambda_{0}<\infty\right)$, the zigzag transition is strongly modified. This is relevant for experiments in annular cells [19,27] and for simulations [14,24]. In these systems, when $\lambda_{0} / d \gg 1$, where $d \equiv L / N$ is the mean interparticle distance, the zigzag transition is still a supercritical pitchfork bifurcation (we assume $N$ is even to allow for a homogeneous zigzag phase in the cell). For smaller values of $\lambda_{0} / d$, the description of the zigzag transition is more complex. As the confinement decreases, the system first evolves from a linear configuration to a homogeneous zigzag. This happens for $\beta=\beta_{Z Z}(\epsilon=0)$. Our previous linear stability analysis allows us to identify the domain of stability of this homogeneous zigzag configuration. This domain extends from $\epsilon=0$ up to a positive $\epsilon>0$ 

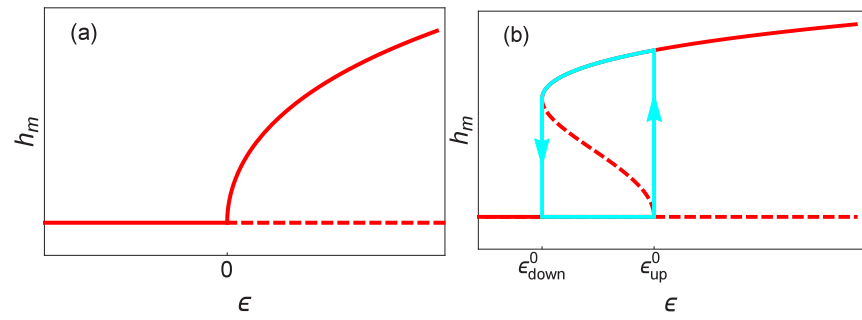

FIG. 1. (a) Supercritical pitchfork bifurcation diagram at $T=$ $0 \mathrm{~K}$. (b) Subcritical pitchfork bifurcation diagram at $T=0 \mathrm{~K}$. The solid red (dark gray) lines correspond to the stable solutions, the dashed red lines to the unstable solutions. The cyan (light gray) lines display an hysteresis loop. The control parameter $\epsilon=1-\beta / \beta_{Z Z}$ is the dimensionless transverse confinement stiffness, which increases from left to right. For the zigzag transition, the order parameter $h_{m}$ is the maximum height of the equilibrium configuration.

that depends on the particle number $N$ at fixed $\lambda_{0} / d$. It is a strongly decreasing function of $N$ and vanishes in the thermodynamic limit $(N \rightarrow \infty$ at fixed $d)$ [14,24]. Then for smaller confinement, an inhomogeneous zigzag pattern is observed in which particles in a line coexist with particles organized in a distorted zigzag (see Fig. 2). We have called this pattern a bubble $[19,24]$. Eventually, at very small confinement $(\epsilon \lesssim 1)$ a homogeneous zigzag is restored.

Since for inhomogeneous patterns the maximum distance $h_{m}$ can be taken as an order parameter, we have performed numerical simulations to determine the variations of $h_{m}(\epsilon)$ for increasing and decreasing $\epsilon$. In Ref. [24], we have provided evidence of hysteretic behavior: The transition from a line toward a bubble, under decreasing confinement, takes place at a smaller confinement than the transition from a bubble toward a line, under increasing confinement. These observations of inhomogeneous equilibrium patterns and of an hysteresis loop all indicate a transition described by a subcritical pitchfork bifurcation [see Fig. 1(b)] for finite range interactions and periodic boundary conditions in the longitudinal direction.

From a theoretical point of view, by considering the vibrational modes of homogeneous patterns in the case of an infinite system of particles, we have shown that $\omega(q=\pi / d)$, the frequency associated with the alternate transverse displacement of neighboring particles, vanishes at the confinement threshold. This means that at the transition, the displacements associated with this soft mode are quenched, resulting in a homogeneous zigzag pattern [14,22]. An infinite system is translationally invariant, so that in the long-wavelength limit the energy of a longitudinal fluctuation vanishes. The translational invariance of the infinite line is thus associated with a second soft mode of vanishing frequency at long wavelength, called a Goldstone mode [28]. Extending the analysis to small nonlinearities, in the thermodynamic limit and near the bifurcation threshold [24], we have exhibited the key role of the nonlinear coupling between the soft mode at the bifurcation and the Goldstone mode. We have obtained the normal form of the bifurcation and the conditions required to observe a supercritical or subcritical pitchfork bifurcation.

In periodic systems, there is also a Goldstone mode linked to the rotational invariance of the periodic cell. We have established that for the parameters used in our experiments and in our simulations, the bifurcation is always subcritical. A quantitative description of the nonlinear localized zigzag patterns (bubbles) observed in the simulations is deduced from the explicit normal form, in excellent agreement with the simulations (see Fig. 2, Appendix B, and Figs. 10-12 of Ref. [24]).

The discussion so far concerns a structural transition observed at temperature $T=0 \mathrm{~K}$. Beyond this first approach, an interesting question is to study the validity and the limits of this description for systems subjected to thermal noise. In this paper, the focus is on this structural transition at finite temperature. Bubble diffusion induced by thermal activation will be presented in a forthcoming paper [29].
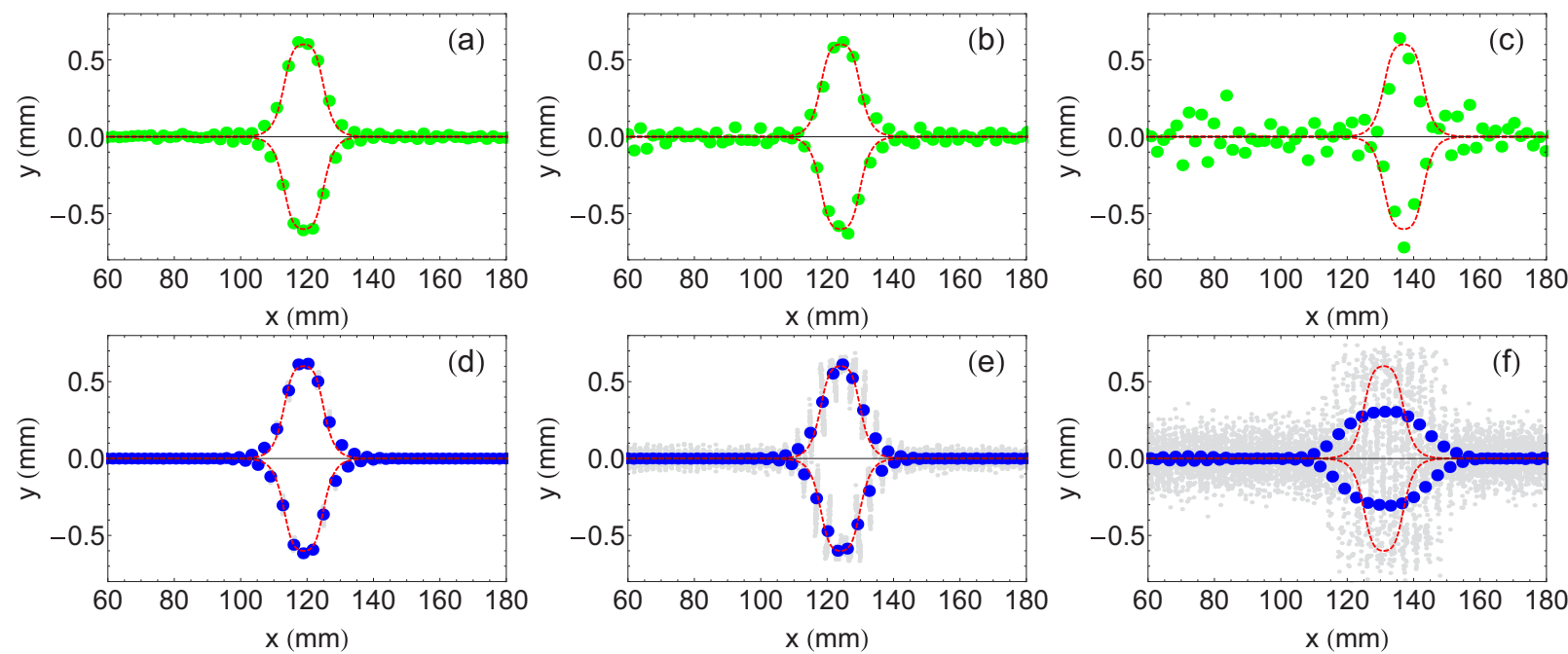

FIG. 2. Particles positions $\left(x_{i}, y_{i}\right)(\mathrm{mm})$ for 128 particles in a bubble configuration $(\epsilon=0.02$ and $d=1.875 \mathrm{~mm})$, for temperatures $T=10^{9}$, $10^{10}$, and $10^{11} \mathrm{~K}$ increasing from left to right. In the top plots, the green dots display instantaneous particle positions and the dashed red line is the theoretical bubble shape. In the bottom plots, the blue (dark gray) dots display the mean values of the positions, the light gray dots display the superposition of all instantaneous positions recorded during the simulation run, and the solid red line is the theoretical bubble shape. 
In the literature, there are some theoretical studies devoted to the thermal effects on supercritical bifurcations [30,31]. Their results are in good agreement with our numerical study of the thermal noise effects on the zigzag transition for longitudinally confined systems [23]. More recently, this approach has been extended to subcritical bifurcations. In particular, it was shown that temperature affects the hysteresis loop [32]. However, the expressions developed in this study are essentially formal, and are seldom compared with actual data.

In this article, we study the effects of white Gaussian additive thermal noise on a subcritical pitchfork bifurcation. The temperature range that we consider is selected according to two limiting constraints. At too low temperature, no effects can be expected since the particles only vibrate around their equilibrium position associated with the configurations obtained at $T=0 \mathrm{~K}$. On the other hand, at too high temperature, the amplitudes of transverse fluctuations are of the same order as the bubble height so that the instantaneous patterns are completely random.

In Sec. II, we first discuss the stability of the bubbles with increasing thermal noise, as well as the relevance of our theoretical description of the bubbles to systems at finite (nonzero) temperature. Then we consider the hysteresis loop between a straight line and a bubble observed at very low temperature [24]. At $T=0 \mathrm{~K}$, the system is in one of the two metastable configurations according to its history: the initial configuration and the direction of change of the control parameter $\epsilon$. It is not the case anymore at $T \neq 0$. Indeed, inside the hysteresis loop the temperature always induces thermal jumps between the two configurations. According to Kramers' theory [33], these jumps are characterized by two transition times $\tau_{L-B}(\epsilon, T)$ (from line to bubble) and $\tau_{B-L}(\epsilon, T)$ (from bubble to line). The existence of these times suggests the important role played in the bifurcation description by the observation time $\tau_{\text {obs }}$ during which $\epsilon$ is constant. As long as $\tau_{\text {obs }}$ is smaller than the characteristic transition times, the jumps between the configurations are not observed. In contrast, the jumps have to be taken into account once one of these Kramers times is smaller than $\tau_{\text {obs }}$. By comparing the three characteristic times $\tau_{\mathrm{obs}}, \tau_{L-B}(\epsilon, T)$, and $\tau_{B-L}(\epsilon, T)$, we may define two distinct regimes: the hysteretic regime for which an hysteresis loop may still be defined, and the intermittent regime for which the thermally activated jumps blur the hysteresis loop. Using the probability density function (PDF) of the instantaneous bubble amplitude $h_{m}(\epsilon, t)$, we are able to obtain an efficient description of the bifurcation at $T \neq 0$. In particular, this enables a detailed study of the relation between $\tau_{\text {obs }}$ and the hysteretic and intermittent regimes. The evolution of the hysteresis loop with temperature and the intermittency effects will be exhibited.

In Sec. III, we focus on a temperature range that is low enough for the hysteresis thresholds to be defined. We show that the hysteresis loop width decreases as the temperature increases. A threshold temperature $T_{c}\left(\tau_{\mathrm{obs}}\right)$ beyond which no hysteresis can be observed is determined as a function of $\tau_{\text {obs }}$. The rough features of this function are recovered with the help of a qualitative analysis. This critical temperature is linked to a particular value $\epsilon_{M}$ of the confinement, which is defined as a Maxwell point for the relevant energy landscape and does not depend on $\tau_{\text {obs. }}$. A numerical value of $\epsilon_{M}$ is provided.

In Sec. IV, we focus on the intermittent regime observed at higher temperature. We show that in this regime a mean residence time for each of the configurations may be defined and measured on the simulations data. The variations of this residence times with temperature can be used to determine the main features of the system energy landscape. We propose a procedure to reconstruct the hysteresis diagram at $T=0 \mathrm{~K}$ from measurements performed at $T \neq 0$. Lastly, we obtain two other numerical values for the confinement $\epsilon_{M}$ with two independent methods, in very good quantitative agreement with the previous one.

The simulation results presented here have been obtained by molecular dynamics with small dissipation in order to emphasize the noise effects on the particle dynamics. The details on the simulations are given in Appendix A. For the sake of completeness, the theoretical description of the bubbles is summarized in Appendix B.

\section{EVOLUTION OF BUBBLE PATTERNS WITH THERMAL NOISE}

\section{A. Bubbles at finite temperature}

At $T=0 \mathrm{~K}$, for each confinement $\epsilon$ corresponding to a bubble state, the particles are organized according to a unique stationary equilibrium pattern. Their shape is accurately deduced from the normal form (B1) given in Appendix B. The theoretical bubble shape is given in Eq. (B3), and the determination of an effective bifurcation parameter, mandatory for finite systems with periodic longitudinal boundary conditions, is made from Eqs. (B5) and (B6), thus without any fitting parameter (see also Ref. [24]). The first question that arises is about the possible persistence of these patterns at higher temperatures and correspondingly the relevance of the theoretical analysis to their description.

In Figs. 2(a)-2(c), we show instantaneous pictures of the periodic cell for a constant transverse confinement and increasing temperatures, which are at least two orders of magnitude higher than those in our previous study [24]. In each case a bubble is evidenced, and regardless of the temperature its shape compares very accurately with the unique theoretical shape deduced from the known value of $\epsilon$. This proves that at these temperatures, these coherent patterns are not destroyed by the thermal fluctuations. To emphasize this point, we plot in Figs. 2(d)-2(f) the superposition of all the positions reached by particles during a typical run. At $T=10^{9}$ and $10^{10} \mathrm{~K}$ [Figs. 2(d) and 2(e)], the bubble shape is basically preserved during the whole simulation run. The bubbles are actually stable equilibrium patterns, the thermal fluctuations only inducing random motions of the particles around their equilibrium positions, with an amplitude that increases with temperature. At higher temperature [ $T=10^{11} \mathrm{~K}$; see Fig. 2(f)], the bubble pattern remains recognizable even if it is almost completely blurred because of the fluctuations.

\section{B. Hysteretic and intermittent regimes}

The systems presenting a subcritical pitchfork bifurcation exhibit a finite range of $\epsilon$ for which the configuration may 
be a line or a bubble (see Appendix B for a brief summary). These metastable configurations are associated with the energy minima $E_{L}(\epsilon)$ for the line and $E_{B}(\epsilon)$ for the bubble. A thermal fluctuation may activate a jump from one configuration to the other at the expense of a maximum of energy $E_{M}(\epsilon)$. We insist on the fact that this maximum is not provided by our continuous model, since knowledge of the full energy landscape requires the complete solution of the discrete $N$-body problem. We discuss this point in detail in Sec. IV.

Let us first consider the system at $T=0 \mathrm{~K}$, and let us look at the configuration evolution when $\epsilon$ increases for a system that is initially in the linear configuration. The system remains in this configuration as long as $E_{L}(\epsilon)$ is a local minimum. When $\epsilon$ reaches the value $\epsilon_{\text {up }}(T=0)$, the minimum $E_{L}\left(\epsilon_{\text {up }}\right)$ and the maximum $E_{M}\left(\epsilon_{\text {up }}\right)$ disappear, and the system jumps to a bubble configuration of smaller energy $E_{B}\left(\epsilon_{\text {up }}\right)$. In the same manner, by considering a system initially in the bubble configuration and a decreasing $\epsilon$, we can define $\epsilon_{\text {down }}(T=0)$. These two thresholds characterize the width $\epsilon_{\text {up }}(T=0)-\epsilon_{\text {down }}(T=0)$ of the hysteresis loop at $T=0 \mathrm{~K}$.

At $T \neq 0 \mathrm{~K}$, these jumps are thermally activated. The jump from a linear to a bubble configuration is characterized by Kramers' activation time $\tau_{L-B}(\epsilon, T) \propto \exp \left[\Delta U_{L-B}(\epsilon) / k_{B} T\right]$, where $\Delta U_{L-B}(\epsilon)=E_{M}(\epsilon)-E_{L}(\epsilon)$, whereas the jump from a bubble to a linear configuration is characterized by $\tau_{B-L}(\epsilon, T) \propto \exp \left[\Delta U_{B-L}(\epsilon) / k_{B} T\right]$, where $\Delta U_{B-L}(\epsilon)=$ $E_{M}(\epsilon)-E_{B}(\epsilon)$. Thus the probability of a jump from one configuration to the other is a function of both $\epsilon$ and $T$.

Because of the existence of these Kramers times, the hysteresis description depends on the observation time $\tau_{\text {obs }}$. For an infinite $\tau_{\mathrm{obs}}$, for any value of $\epsilon$, the Kramers times are necessarily smaller than $\tau_{\text {obs }}$, and both configurations are observed, independently of the initial state. Therefore, we cannot define the transition thresholds as for $T=0 \mathrm{~K}$, and the hysteresis loop is meaningless. However, in practice, any actual numerical or experimental observation implies a finite $\tau_{\text {obs }}$. The hysteresis thresholds have to be redefined and depend on this observation time. A threshold can be defined if a jump from an initial configuration to the other one occurs during the observation time, while the reverse jump does not occur because the corresponding Kramers time is too long with respect to $\tau_{\mathrm{obs}}$. In this case, speaking of an hysteresis loop that depends on the temperature makes sense. In contrast, when the characteristic times for both jumps are smaller than $\tau_{\mathrm{obs}}$, the two configurations are observed during $\tau_{\text {obs }}$, regardless of the initial configuration of the system. The memory of the initial configuration is not conserved, so that no hysteresis loop can be defined. We will speak in this case of an intermittent regime.

\section{Definition of an order parameter in the presence of thermal noise}

To describe the transition, it is mandatory to define the order parameter $h_{m}(\epsilon)$ for each confinement $\epsilon$ that characterizes accurately and unambiguously the configurations explored by the system. The definition of this parameter at finite temperature is not obvious and requires an adapted procedure.

The simulations only provide instantaneous configurations. Such configurations are plotted for three different temperatures in Figs. 2(a)-2(c). They are very sensitive to the thermal fluctuations, however they are all in close agreement with the same theoretical description, independently of the temperature, as shown in Sec. II A. Thus, the main question is how to determine an amplitude $h_{m}(\epsilon)$ that could well characterize this configuration. We could be tempted to define $h_{m}(\epsilon)$ as the maximum distance between the particles and the longitudinal axis, measured from time-averaged configurations. This procedure makes sense at low temperature, but it introduces important errors at high temperature. Indeed, at small temperature, we see that the averaged configurations [see Figs. 2(d) and 2(e)] are very close to the instantaneous ones [see Figs. 2(a) and 2(b)]. In contrast, the averaged configuration at the highest temperature, shown in Fig. 2(f), has nothing in common with the instantaneous configuration of Fig. 2(c). Thus the strong discrepancy between the averaged configuration of Fig. 2(f) and those of Figs. 2(d) and 2(e) is a spurious effect that prevents $h_{m}(\epsilon)$ from being defined in the averaged configuration.

We have therefore developed a consistent process to determine the amplitude $h_{m}(\epsilon)$ at every temperature. To take advantage of the improvement in the statistics implied by the duration of the whole simulation, while avoiding averaging artifacts, we define $h_{m}(\epsilon, t)$ as the maximum distance between the particles and the cell axis at time $t$, and we consider its probability density function (PDF). The PDFs are shown in Fig. 3 for two temperatures and increasing values of $\epsilon$ near the bifurcation threshold.

The position of the maximum of this distribution may be used as an order parameter. Let us first consider a strong confinement $\epsilon$ for which the particles are along a straight line at zero temperature, so that $h_{m}(\epsilon, T=0, t)=0$ and the PDF is the Dirac $\delta$ function $\delta(h)$. At finite temperature, the maximum height $h_{m}(\epsilon, T>0, t)$ is strictly positive at each time and increases with temperature [see Fig. 3(a)]. Thus the corresponding PDF is slightly shifted above zero and is broadened around the most probable value $h_{m}(\epsilon, T>0)$. Note that from now on we emphasize the dependence of $h_{m}$ upon the temperature.

For a confinement such that the stable pattern at $T=0$ is a bubble, the PDF is a Dirac $\delta$ function centered on a strictly positive value that is the bubble amplitude. At finite temperature, the PDF evidences a small shift toward higher values and a broadening, as was the case for the straight line [see Fig. 3(c)]. The configuration is nevertheless easy to distinguish from a straight line, because despite the thermal fluctuations, the most probable value $h_{m}(\epsilon, T>0)$ is much higher than that in the previous case.

Lastly, let us consider a confinement $\epsilon$ in the hysteretic domain at $T=0 \mathrm{~K}$. At zero temperature, the distribution of $h_{m}(\epsilon, T=0, t)$ corresponds to Dirac $\delta$ functions centered around $h_{m}=0$ if the initial configuration was a straight line or around $h_{m}>0$ if the initial configuration was a bubble configuration. The PDF of $h_{m}(\epsilon, T, t)$ at finite temperature is shown in Fig. 3(b). At low temperature $\left[T=10^{10} \mathrm{~K}\right.$, cyan (light gray) symbols], for which no jump between the two allowed configurations may be expected, the system simply keeps a memory of its initial configuration and explores the configuration space around only one stable attractor. Depending upon the initial condition, the PDF is a finite width distribution centered around a small value of $h_{m}$ for 

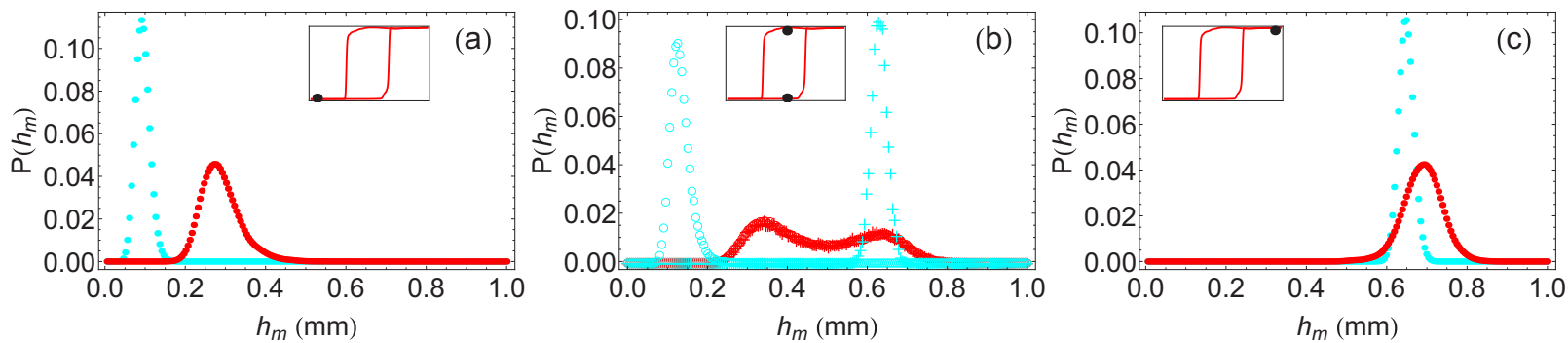

FIG. 3. PDF of the height $h_{m}(\epsilon, T, t)$ (in mm) for a system of 128 particles for three different confinements: (a) $\epsilon=-0.05$, (b) $\epsilon=0.001$, and (c) $\epsilon=+0.05$. The red (dark gray) and cyan (light gray) dots correspond, respectively, to $T=10^{10}$ and $10^{11} \mathrm{~K}$. In the inset of each plot, we show the corresponding point in the hysteresis loop measured at a very low temperature $T=10^{7} \mathrm{~K}$. In (b) the open circles correspond to an initial condition that is a line, and the crosses correspond to an initial condition that is a bubble.

the fluctuating straight line [Fig. 3(b), cyan circles] or around a much larger value of $h_{m}$ for the fluctuating bubble [Fig. 3(b), cyan crosses]. At higher temperature $\left[T=10^{11} \mathrm{~K}\right.$, red (dark gray) symbols], there are thermally activated jumps between the line and the bubble. This is the intermittent regime, for which the memory of its initial condition is lost by the system. The PDF evidences a camelback shape [see Fig. 3(b), red (dark gray) symbols]. The first peak corresponds to the fluctuating straight-line configurations, and the second peak corresponds to the fluctuating bubble configurations. Both peaks are broadened because of the thermal fluctuations, and the probability to
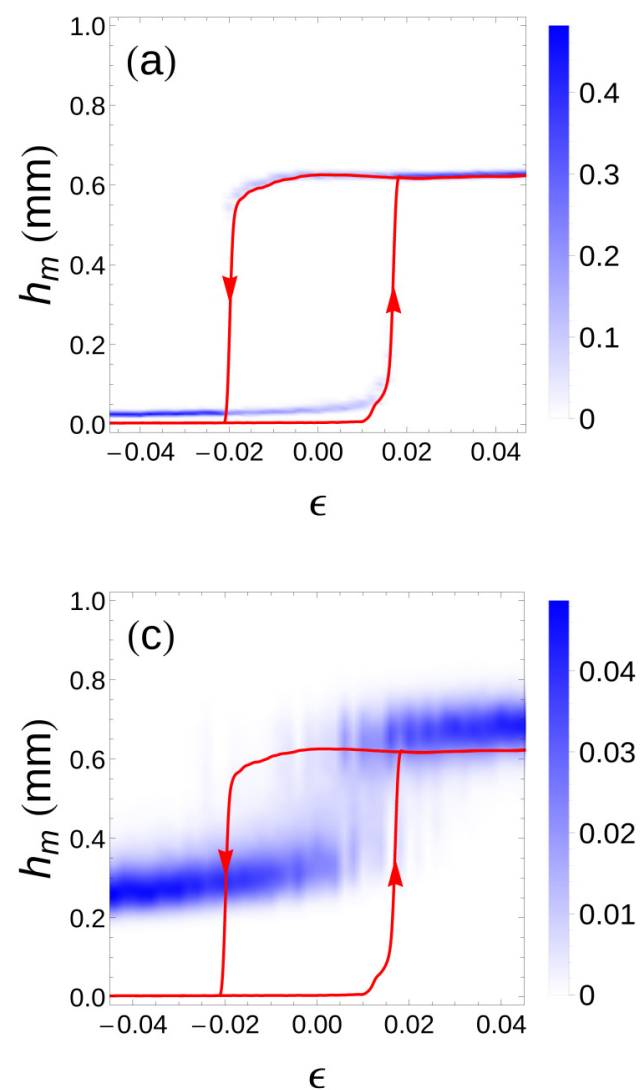

observe an intermediate configuration that cannot be described either as a line or as a bubble is strictly positive.

\section{Evolution of the hysteresis with temperature}

The thermal effects on the hysteresis diagram can be studied by following the evolution of the PDFs of $h_{m}(\epsilon, T, t)$ with the confinement $\epsilon$ for several temperatures. In Fig. 4, we display the reconstructed hysteresis diagram with the help of the PDFs, shown as density plots for each confinement $\epsilon$.

For low temperature, the thermal energy is insufficient to induce intermittency during a step at constant $\epsilon$ of duration
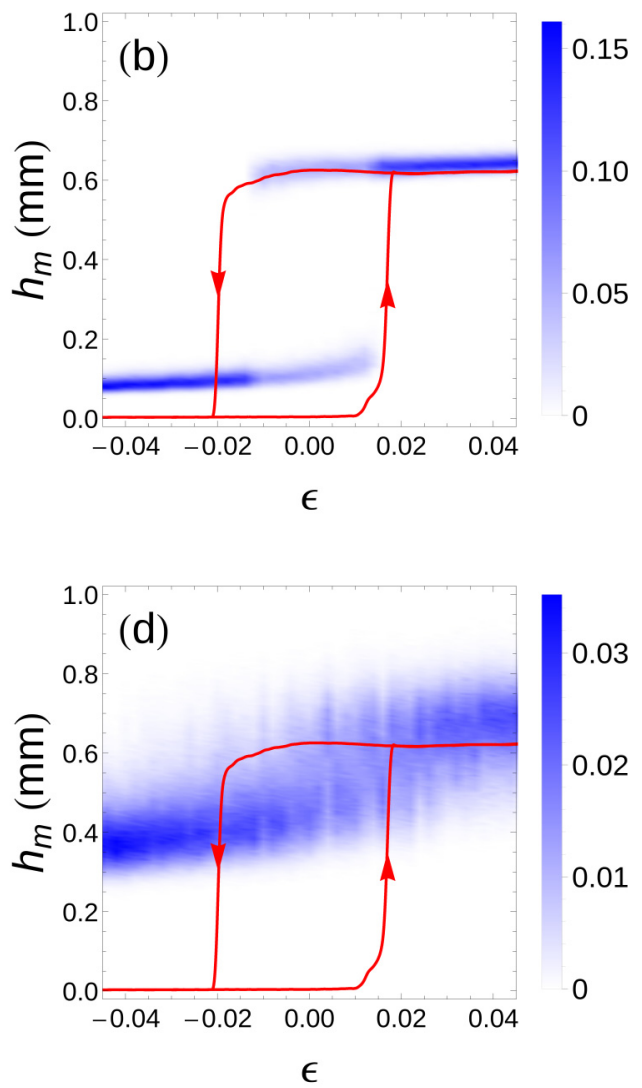

FIG. 4. Hysteresis diagrams reconstructed from the PDF of $h_{m}(\epsilon, T, t)$, measured at temperatures $T=10^{9} \mathrm{~K}(\mathrm{a}), T=10^{10} \mathrm{~K}(\mathrm{~b}), T=10^{11} \mathrm{~K}$ (c), and $T=2 \times 10^{11} \mathrm{~K}(\mathrm{~d})$. The solid red line represents the hysteresis loop obtained at very low temperature $T=10^{7} \mathrm{~K}$. The PDFs are displayed as density plots for each $\epsilon$ and correspond to the blue scales on the right. For $T=10^{9}$ and $10^{10} \mathrm{~K}$, the system keeps the memory of its initial condition, and the evolution of $\epsilon$ during the whole simulation corresponds to the red arrows. At $T=10^{11}$ and $2 \times 10^{11} \mathrm{~K}$, all memory is lost by the system, and the diagrams do not depend on the evolution of $\epsilon$ during the whole simulation. 
$\tau_{\text {obs. }}$. The hysteresis loop is still observed, and the diagram is only slightly modified with respect to the case of the very low temperature $T=10^{7} \mathrm{~K}$, as shown in Figs. 4(a) and 4(b). The comparison between the hysteresis diagrams obtained at $T=10^{9}$ and $10^{10} \mathrm{~K}$ [Figs. 4(a) and 4(b), respectively] shows that its width decreases as the temperature increases. This point will be discussed in detail in Sec. III.

For higher temperature, the intermittent behavior strongly modifies the transition diagram, as shown in Figs. 4(c) and 4(d). Now the thermally activated jumps between the linear configuration and the bubble happen in the range $\epsilon_{\text {down }}(T=$ $0)<\epsilon<\epsilon_{\text {up }}(T=0)$. At $T=10^{11}$ and $2 \times 10^{11} \mathrm{~K}$, during a step at constant $\epsilon$ of duration $\tau_{\mathrm{obs}}$, the system loses all memory of its initial state, and the hysteresis loop is no longer observed.

It is possible to get a qualitative picture of these hysteresis diagram. Whereas at $T=0 \mathrm{~K}$ the energy of the system is either $E_{L}(\epsilon)$ or $E_{B}(\epsilon)$, the thermal agitation results in an exploration of the neighborhoods of $E_{L}(\epsilon)$ and $E_{B}(\epsilon)$ by the energy of the system. This induces a broadening of the PDF of $h_{m}(\epsilon, T, t)$. As mentioned before, we do not know the complete energy landscape. We may nevertheless obtain a rough estimate of the distribution of $h_{m}(\epsilon, T, t)$ by considering only homogeneous configurations. In this simpler model, the expression of the energy reads

$$
E(\epsilon, h)=\frac{\epsilon \beta_{Z Z}}{2} h^{2}+\frac{g_{3}}{4} h^{4}-\frac{g_{5}}{6} h^{6},
$$

where $\epsilon$ is the order parameter, $h$ is the observable (the amplitude of the homogeneous zigzag), and $\beta_{Z Z}$ and $g_{i}$ are positive constants [see Eq. (B1) of Appendix B]. The hysteresis loop at $T=0$ is then characterized by the existence of two local energetic minima, one for $h=0$ denoted $E_{0}(\epsilon)$ and one for $h \neq 0$ denoted $E_{1}(\epsilon)$. To exhibit the broadening, we may estimate the extension of $h$ reached by the system thanks to the thermal fluctuations by numerically extracting $h$ from the equations $E(\epsilon, h)=E_{0}(\epsilon)+k_{B} T$ near the solution $h(T=0)=0$ and $E(\epsilon, h)=E_{1}(\epsilon)+k_{B} T$ near the solution $h(T=0) \neq 0$. The domains explored by $h$ calculated using this procedure are plotted in Fig. 5 when an hysteresis loop is observed at low temperature [Fig. 5(a)], and when an intermittent regime is observed at high temperature [Fig. 5(b)]. They display the same qualitative features as those obtained in the simulations for inhomogeneous configurations (Fig. 4).

\section{EFFECT OF THERMAL NOISE ON THE HYSTERESIS THRESHOLDS}

We now examine in detail the influence of thermal noise on the hysteresis loop for a given observation time $\tau_{\text {obs }}$ during which $\epsilon$ is kept constant.

At $T=0 \mathrm{~K}$, the thresholds $\epsilon_{\text {down }}(T=0)$ and $\epsilon_{\text {up }}(T=0)$ only depend on the stability of the equilibrium configurations. At finite temperature, the thresholds $\epsilon_{\text {down }}\left(T, \tau_{\text {obs }}\right)$ and $\epsilon_{\text {up }}\left(T, \tau_{\text {obs }}\right)$ also depend on the temperature and on the observation time. Let us first consider a system initially in the linear configuration and increase $\epsilon$ by successive steps of duration $\tau_{\mathrm{obs}}$. For the $i$ th step, $\epsilon=\epsilon_{i}$, the system stays in a straight-line configuration as long as Kramers' transition time $\tau_{L-B}\left(\epsilon_{i}, T\right)>\tau_{\mathrm{obs}}$, because its initial configuration at the step $(i-1)$ is a line, and $\tau_{\text {obs }}$ is insufficient for the transition
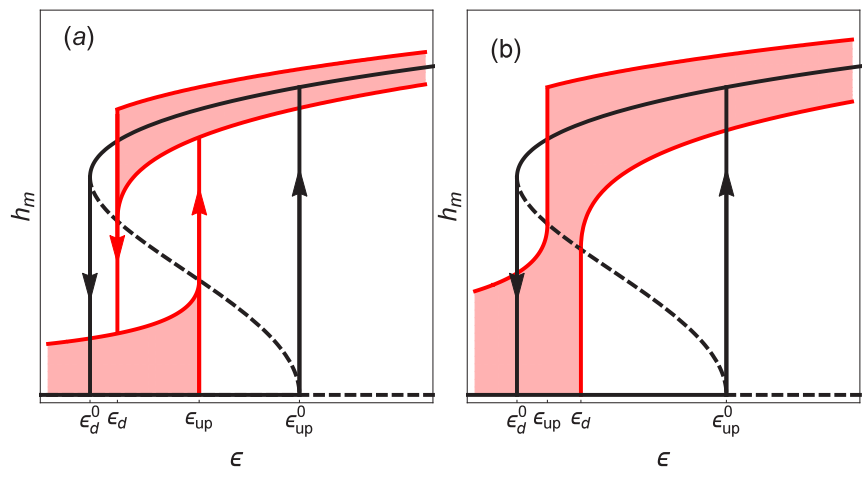

FIG. 5. Qualitative estimate of the thermal broadening for a subcritical pitchfork bifurcation. For each $\epsilon$ the width of the red (gray) shaded area quantifies the reachable values of $h$. The solid black lines display the stable homogeneous states, the dashed black lines the unstable states, and the black arrows the hysteresis loop at $T=0$. (a) At low temperature, the two explored regions corresponding to the two equilibrium states are always distinct, and the hysteresis loop remains as shown by the red arrows. This should be compared to Figs. 4(a) and 4(b). (b) At high temperature, the two explored areas merge and the hysteresis loop is blurred. This should be compared to Figs. 4(c) and 4(d).

to happen. The transition toward the bubble configuration takes places when $\tau_{L-B}\left(\epsilon_{i}, T\right) \sim \tau_{\text {obs }}$ since the observation of a jump toward the bubble configuration during $\tau_{\mathrm{obs}}$ is now more likely. Once the transition is achieved, if $\tau_{B-L}\left(\epsilon_{i}, T\right)>$ $\tau_{\text {obs }}$, the system remains in the bubble configuration. Thus $\tau_{L-B}\left(\epsilon_{\mathrm{up}}, T\right) \sim \tau_{\mathrm{obs}}$ defines the hysteresis threshold $\epsilon_{\mathrm{up}}\left(T, \tau_{\mathrm{obs}}\right)$ providing that $\tau_{B-L}\left(\epsilon_{\mathrm{up}}, T\right)>\tau_{\mathrm{obs}}$ at temperature $T$. Note that since $\tau_{L-B}(\epsilon, T) \propto \exp \left[\Delta U_{L-B}(\epsilon) / k_{B} T\right]$ decreases with temperature, the condition $\tau_{L-B}\left(T, \epsilon_{\text {up }}\right) \sim \tau_{\text {obs }}$ implies that $\epsilon_{\mathrm{up}}\left(T, \tau_{\mathrm{obs}}\right)$ is a decreasing function of the temperature. Similarly, following the evolution of an initial bubble configuration as $\epsilon$ decreases, we can define the threshold $\epsilon_{\text {down }}\left(T, \tau_{\text {obs }}\right)$ [with $\tau_{B-L}\left(\epsilon_{\text {down }}, T\right) \sim \tau_{\text {obs }}$ and $\tau_{L-B}\left(\epsilon_{\text {down }}, T\right)>\tau_{\text {obs }}$ ]. In the opposite way, this threshold increases with temperature.

Figure 6 represents $\epsilon_{\text {down }}\left(T, \tau_{\text {obs }}\right)$ and $\epsilon_{\text {up }}\left(T, \tau_{\text {obs }}\right)$ as functions of $T$ from our simulation data. The variations of $\epsilon_{\text {down }}\left(T, \tau_{\text {obs }}\right)$ and $\epsilon_{\text {up }}\left(T, \tau_{\text {obs }}\right)$ with temperature agree with the above analysis. The width of the hysteresis loop, $\epsilon_{\text {up }}\left(T, \tau_{\text {obs }}\right)-$ $\epsilon_{\text {down }}\left(T, \tau_{\text {obs }}\right)$, eventually vanishes at a threshold temperature

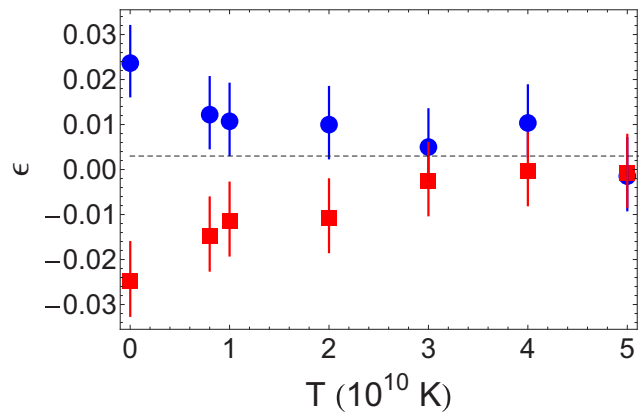

FIG. 6. Evolution of the thresholds $\epsilon_{\mathrm{up}}\left(T, \tau_{\mathrm{obs}}\right)$ (blue dots) and $\epsilon_{\text {down }}\left(T, \tau_{\text {obs }}\right)$ (red squares) with temperature. 
$T_{c}\left(\tau_{\text {obs }}\right)$. This temperature is defined by $\tau_{L-B}\left(\epsilon_{\text {down }}, T_{c}\right) \sim$ $\tau_{B-L}\left(\epsilon_{\mathrm{up}}, T_{c}\right) \sim \tau_{\mathrm{obs}}$.

A qualitative picture of the variation of $T_{c}$ with $\tau_{\mathrm{obs}}$ may be obtained. For an infinite observation time, intermittency is always present, thus no hysteresis loop can be defined at finite temperature so that $T_{c}\left(\tau_{\mathrm{obs}} \rightarrow \infty\right) \rightarrow 0$. To analyze the variation of $T_{c}\left(\tau_{\mathrm{obs}}\right)$ for finite $\tau_{\mathrm{obs}}$, we may derive the evolution of $\epsilon_{\text {down }}\left(T, \tau_{\text {obs }}\right)$ and $\epsilon_{\mathrm{up}}\left(T, \tau_{\mathrm{obs}}\right)$ from the following two equations:

$$
\begin{gathered}
k_{B} T \ln \left(\frac{\tau_{\mathrm{obs}}}{\tau_{0}}\right)=\Delta U_{L-B}\left(\epsilon_{\mathrm{up}}\right), \\
k_{B} T \ln \left(\frac{\tau_{\mathrm{obs}}}{\tau_{0}}\right)=\Delta U_{B-L}\left(\epsilon_{\mathrm{down}}\right),
\end{gathered}
$$

where for the sake of simplicity the characteristic time $\tau_{0}$ introduced by Kramers [33] may be considered constant.

As of now these equations are only formal because we do not know the energy gaps. As before, we get a qualitative interpretation by considering homogeneous patterns. We show in Fig. 7 the evolution of $\epsilon_{\text {up }}\left(T, \tau_{\mathrm{obs}}\right)$ and $\epsilon_{\text {down }}\left(T, \tau_{\mathrm{obs}}\right)$ calculated for three ratios $\tau_{\text {obs }} / \tau_{0}$. They are obtained by directly solving Eqs. (2) and (3), where the unknown energetic barriers $\Delta U_{L-B}$ and $\Delta U_{B-L}$ are estimated for homogeneous patterns from the simple energetic expression of Eq. (1). We recover the qualitative features of the functions $\epsilon_{\text {down }}\left(T, \tau_{\text {obs }}\right)$ and $\epsilon_{\text {up }}\left(T, \tau_{\text {obs }}\right)$ measured from the simulations data, as shown in Fig. 6. This is also consistent with simulation results presented by Agez et al. [32]; see their Fig. 15.

The crossing of the plots $\epsilon_{\text {down }}\left(T, \tau_{\text {obs }}\right)$ and $\epsilon_{\text {up }}\left(T, \tau_{\text {obs }}\right)$ happens by construction for $T=T_{c}\left(\tau_{\mathrm{obs}}\right)$. It takes place at a confinement $\epsilon=\epsilon_{M}$, which does not depend on $\tau_{\mathrm{obs}}$. This particular value $\epsilon_{M}$ is defined as the confinement for which both energetic barriers are equal:

$$
\Delta U_{L-B}\left(\epsilon_{M}\right)=\Delta U_{B-L}\left(\epsilon_{M}\right),
$$

which suggests that it should be called the Maxwell point. Note that this equation and thus the value of $\epsilon_{M}$ are independent of temperature. As shown in Fig. 6, the value of $\epsilon_{M}$ is $0 \pm 0.01$.

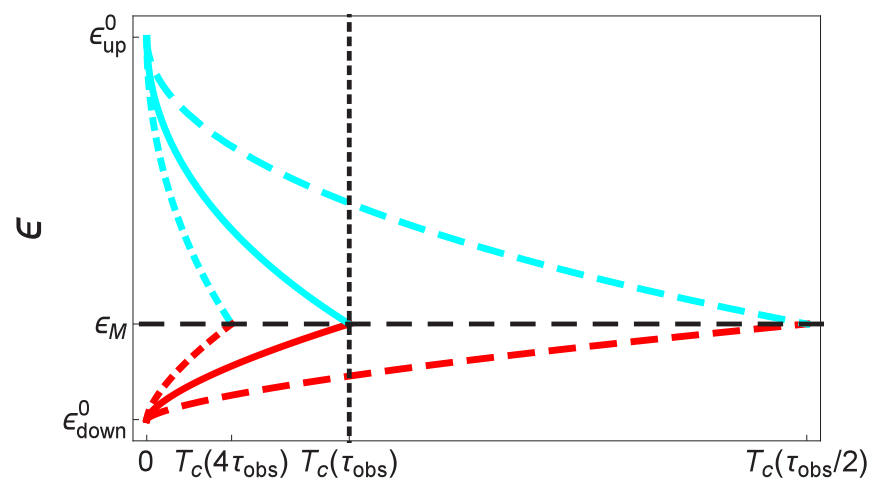

FIG. 7. Theoretical variation of the thresholds $\epsilon_{\mathrm{up}}\left(T, \tau_{\mathrm{obs}}\right)$ (cyan, light gray) and $\epsilon_{\text {down }}\left(T, \tau_{\mathrm{obs}}\right)$ (red, dark gray) with the temperature, for observation times $\tau_{\mathrm{obs}} / \tau_{0}=0.5$ (dashed line), $\tau_{\mathrm{obs}} / \tau_{0}=1$ (solid line), and $\tau_{\mathrm{obs}} / \tau_{0}=4$ (dotted line). The critical temperature $T_{c}\left(\tau_{\mathrm{obs}}\right)$ decreases as $\tau_{\text {obs }}$ increases. The confinement $\epsilon_{M}$ corresponds to the Maxwell point at which both stable states have the same energy.

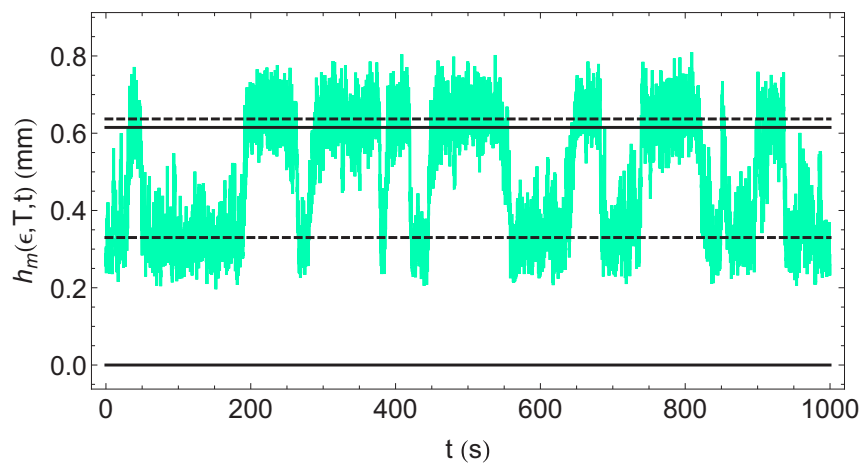

FIG. 8. Instantaneous maximum amplitude $h_{m}(\epsilon, T, t)$ (in $\mathrm{mm}$ ) as a function of time $t$ (in s) for $\epsilon=0.005$ with $T=8 \times 10^{10} \mathrm{~K}$. The solid black lines indicate the amplitudes $h_{m}(\epsilon, 0, t)$ for the linear and bubble configurations at $T=0 \mathrm{~K}$, and the dashed black lines indicate the time-averaged amplitudes $h_{m}(\epsilon, T)$ for the linear and bubble configurations at $T=8 \times 10^{10} \mathrm{~K}$.

\section{INTERMITTENT REGIME AND ENERGY LANDSCAPE}

Let us now consider the intermittent regime. Despite the large fluctuations induced by the thermal agitation, we have taken advantage of the unique opportunity given by the numerous transitions between configurations to determine the main features of the energy landscape (Maxwell point and energetic barrier height).

\section{A. Residence time and energy landscape}

For temperature higher than $T_{c}\left(\tau_{\mathrm{obs}}\right)$, the intermittent regime is reached as soon as $\tau_{B-L}(\epsilon, T)<\tau_{\text {obs }}$ and $\tau_{L-B}(\epsilon, T)<\tau_{\text {obs }}$. An example of such intermittency is shown in Fig. 8, which presents a typical time evolution of the maximum height $h_{m}(\epsilon, T, t)$. The system stays for a finite time in one configuration and jumps quickly to the other configuration. Let $\tau_{L}$ be the time spent by the system in the straight line configuration, and $\tau_{B}$ is the time spent by the system in the bubble configuration. The PDFs of these times are widely distributed, as shown in Figs. 9(a) and 9(b).

From such distributions, the mean residence times $\left\langle\tau_{L}\right\rangle$ and $\left\langle\tau_{B}\right\rangle$ may be calculated [34]. To check the relevance of these two times, the PDFs obtained from simulations have to be compared to the homogeneous Poisson probability density $P(\tau)=e^{-\tau /\langle\tau\rangle} /\langle\tau\rangle$, which describes the probability distribution of residence times that are random and of small probability [33]. In Figs. 9(a) and 9(b), we show that the PDF measured from the simulations is very well fitted by the expected probability distribution.

The knowledge of these mean residence times provides a lot of useful information. Obviously, it gives the most likely state of the system, which cannot be deduced from the transition diagram blurred at this high temperature [see, e.g., Figs. 4(c) and 4(d)]. Moreover, the evolution of these residence times with the confinement $\epsilon$ gives another method to determine the Maxwell point. At the Maxwell point, we expect that $\left\langle\tau_{L}\right\rangle=\left\langle\tau_{B}\right\rangle$. We display in Fig. 9(c) the evolution of the mean residence times $\left\langle\tau_{L}\right\rangle$ and $\left\langle\tau_{B}\right\rangle$ as a function of $\epsilon$ at a temperature $T=8 \times 10^{10} \mathrm{~K}$. The two mean residence times are equal for $\epsilon_{M}=0.003 \pm 0.004$, which is in very good agreement with 

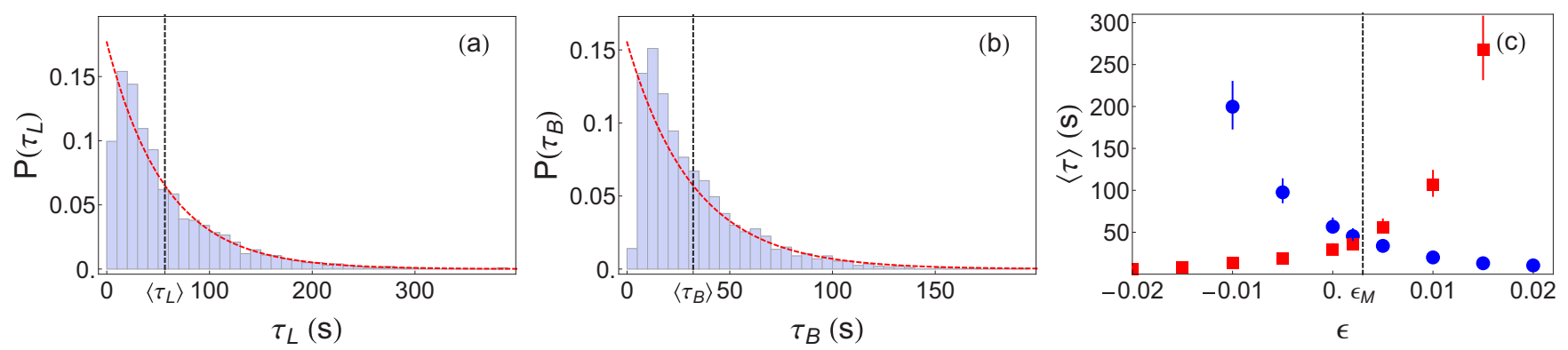

FIG. 9. (a) Distribution $P\left(\tau_{L}\right)$ of the residence times $\tau_{L}$ (in s) for a line. (b) Distribution $P\left(\tau_{B}\right)$ of the residence times $\tau_{B}$ (in s) for a bubble. In both cases, the confinement is $\epsilon=0.0$ and the temperature is $T=8 \times 10^{10} \mathrm{~K}$. The red dashed line is a fit with the expected Poisson distribution, $P(\tau)=e^{-\tau /\langle\tau\rangle} /\langle\tau\rangle$, from which we deduce the relevant mean residence times $\langle\tau\rangle$ that are indicated by the black dashed lines. (c) Plots of the mean residence times $\left\langle\tau_{L}\right\rangle$ (blue dots) and $\left\langle\tau_{B}\right\rangle$ (red squares) as a function of $\epsilon$ at $T=8 \times 10^{10} \mathrm{~K}$. The crossing of these plots defines the Maxwell point $\epsilon_{M}$ (dotted line).

our previous estimate deduced from the zero width of the hysteretic loop (see Fig. 6).

Furthermore, the energy barriers $\Delta U_{L-B}(\epsilon)$ and $\Delta U_{B-L}(\epsilon)$ for a given $\epsilon$ may be estimated from the evolution of residence times with temperature. The first step is to determine the evolution of the mean residence times with the temperature at fixed $\epsilon$ (see Fig. 10). The plots measured on the simulation data are very well fitted by a Kramers law [35], which gives a reliable estimate for the energy barriers $\Delta U_{L-B}(\epsilon)$ and $\Delta U_{B-L}(\epsilon)$ for a given $\epsilon$.

When we repeat this process for several values of $\epsilon$, we are able to plot the evolution of the energy barriers with the confinement $\epsilon$, as shown in Fig. 11. As expected, these barriers are monotonic functions of $\epsilon$, with $\Delta U_{L-B}(\epsilon)$ decreasing and $\Delta U_{B-L}(\epsilon)$ increasing. Note that these energetic barriers are independent of the temperature and observation time, as expected for these intrinsic parameters of the system. The crossing point of these two curves, for which the two barriers are equal, characterizes by definition the Maxwell point $\epsilon_{M}$ [see Eq. (4)]. Its value measured in Fig. 11 is $\epsilon_{M}=0.007 \pm$ 0.004 . Let us emphasize that our three independent estimates of $\epsilon_{M}$ are fully consistent. Note also that this procedure is useful since the complete energy landscape is unknown and since the Maxwell point cannot be directly deduced from a single hysteresis diagram.

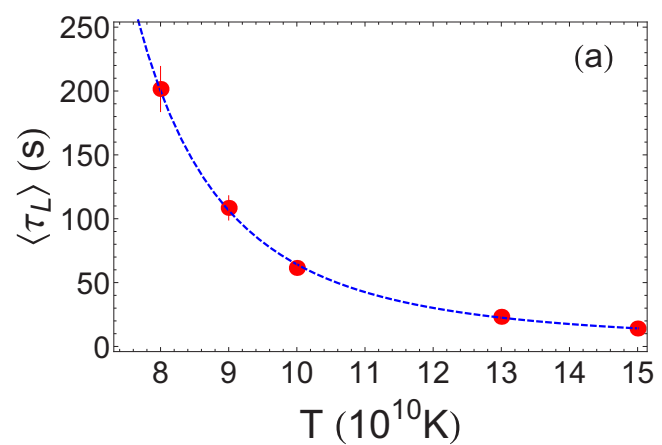

\section{B. Reconstruction of the hysteresis loop at $T=0 \mathrm{~K}$}

Another important point is that we are able to recover the hysteresis loop at $T=0 \mathrm{~K}$ from the data recorded at $T \neq 0$. As shown by Fig. 11(a), the energetic barriers are roughly linear functions of the confinement $\epsilon$. We can thus extrapolate the data to estimate the values of $\epsilon_{\text {up }}(T=0)$ and $\epsilon_{\text {down }}(T=0)$ for which the energetic barriers $\Delta U_{L-B}(\epsilon)$ and $\Delta U_{B-L}(\epsilon)$, respectively, vanish. These values correspond by definition to the thresholds and give the width of the hysteresis loop at $T=0 \mathrm{~K}$. Moreover, the upper branch of the hysteresis loop is quite accurately given by the position of the maximum of a typical PDF since it varies slowly with temperature, while the lower branch is zero by definition of the line configuration. In Fig. 11(b) we show an hysteresis loop at $T=0 \mathrm{~K}$, reconstructed from measurements at $T \neq 0$, that compares rather well with the hysteresis loop evidenced by very-low-temperature simulations.

To conclude this discussion, let us emphasize an interesting opportunity given by our analysis: We are able to obtain the local maximum $E_{M}(\epsilon)$ in the otherwise unreachable energy landscape. This is schematically illustrated in Fig. 12(a). The energy $E_{L}(\epsilon)$ of a line and the energy $E_{B}(\epsilon)$ of a bubble may be calculated from the equilibrium configurations obtained in the simulations, and $E_{M}(\epsilon)$ is the energy amount that has to be spent in order to jump between the line and bubble

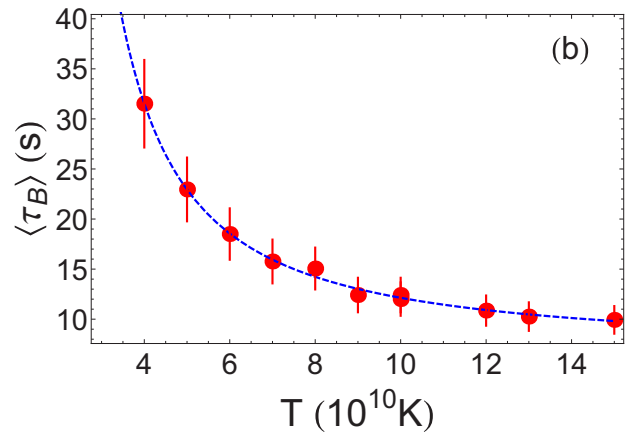

FIG. 10. (a) Plot of the mean residence time $\left\langle\tau_{L}\right\rangle$ (in s) for a line, as a function of temperature (in $10^{10} \mathrm{~K}$ ), for $\epsilon=-0.01$. (b) Plot of the mean residence time $\left\langle\tau_{B}\right\rangle$ (in s) for a bubble, as a function of temperature (in $10^{10} \mathrm{~K}$ ), for $\epsilon=-0.01$. The red dots are simulation data, and the dashed blue line is the fit performed with Kramers' law. 

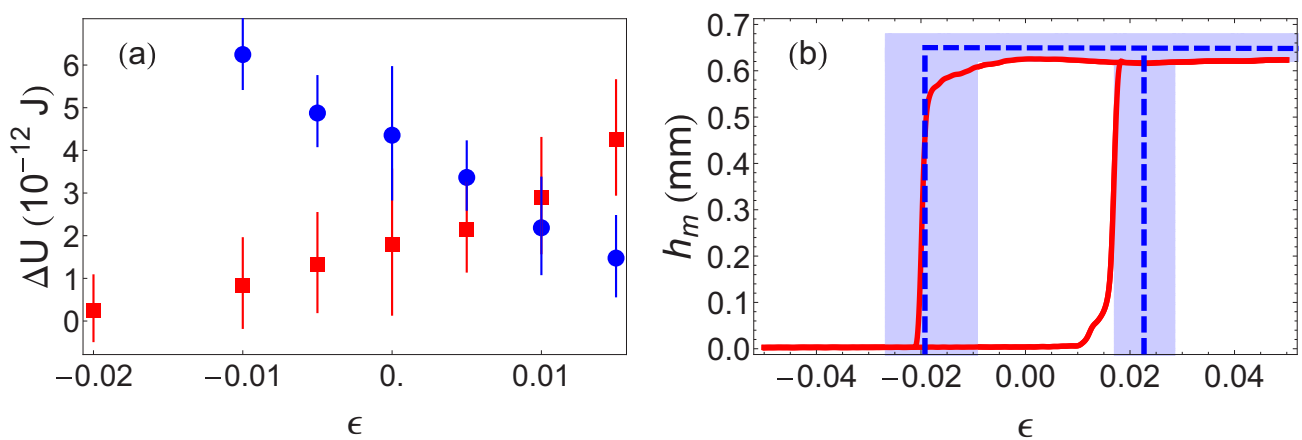

FIG. 11. (a) Plot of the energetic barriers $\left(10^{-12} \mathrm{~J}\right)$ as functions of the confinement parameter $\epsilon$, for a transition from a line to a bubble $\left[\Delta U_{L-B}(\epsilon)\right.$, blue dots] and from a bubble to a line $\left[\Delta U_{B-L}(\epsilon)\right.$, red squares]. (b) The solid red line displays the hysteresis loop obtained at very low temperature $\left(T=10^{7} \mathrm{~K}\right)$. The dashed blue lines are determined from the extrapolation of the energetic barrier plots (see text for details). The shaded area is a measure of the uncertainty.

configuration. Notice that we know neither the value of $E_{M}(\epsilon)$ nor the corresponding configuration. Nevertheless, we may write

$$
E_{M}(\epsilon)=E_{L}(\epsilon)+\Delta U_{L-B}(\epsilon)=E_{B}(\epsilon)+\Delta U_{B-L}(\epsilon) .
$$

We plot in Fig. 12(b) these two sums. They are in very good agreement, especially if we consider the small values of the energetic barriers with respect to the configuration energies, which are of the order of $15 \mathrm{~nJ}$. This result supports our analysis of the residence times as Kramers times. Therefore, we propose this explicit procedure to determine the main features of the energy landscape in such many-body systems [36].

\section{CONCLUSION}

The subcritical pitchfork bifurcation studied in this paper is the zigzag transition in systems of particles that are transversally confined, with short-range (non-Coulombic) interactions and periodic boundary conditions in the longitudinal direction. As a consequence of the subcriticality, there is a finite range of transverse confinement stiffnesses for which the stable configuration at zero temperature is a bubble, where a localized zigzag pattern takes place in a finite part of the periodic cell.
We have shown that these configurations remain stable for a wide temperature range.

At zero temperature, the transition between a straight line, for which all particles are aligned along the bottom of the transverse confinement potential, and a bubble is hysteretic. We have studied the influence of thermal noise on this hysteresis loop. Its description is more difficult than at $T=0 \mathrm{~K}$ since thermally activated jumps between the two configurations always occur and the system can never stay forever in a unique metastable state. But since an actual simulation lasts a finite time, these jumps need to be taken into account only if their characteristic Kramers times, $\tau_{L-B}(\epsilon, T)$ and $\tau_{B-L}(\epsilon, T)$, are smaller than the observation time $\tau_{\text {obs }}$. Because the Kramers times are temperature-dependent, two different regimes have to be considered according to the temperature value with respect to a critical temperature $T_{c}\left(\tau_{\mathrm{obs}}\right)$ such that $\tau_{L-B}\left(\epsilon, T_{c}\right) \sim \tau_{B-L}\left(\epsilon, T_{c}\right) \sim \tau_{\text {obs }}$. For $T<T_{c}\left(\tau_{\text {obs }}\right)$, the duration of a simulation is short enough for the system to keep the memory of its initial condition. An hysteresis loop is still observed, with a width that decreases as the temperature increases toward $T_{c}\left(\tau_{\mathrm{obs}}\right)$. For $T>T_{c}\left(\tau_{\mathrm{obs}}\right)$, the memory of the initial condition is lost due to stochastic jumps between the linear and bubble configurations evidenced on the duration $\tau_{\mathrm{obs}}$, and the hysteresis loop is no longer seen.
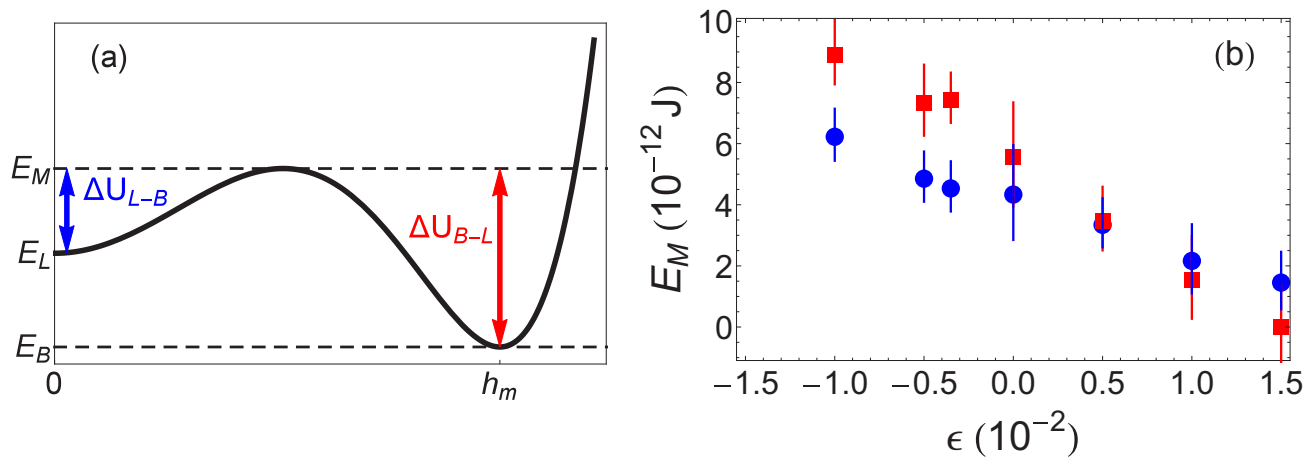

FIG. 12. (a) Schematic representation at a given $\epsilon$ of the energy landscape, where the arrows indicate the energetic barriers $\Delta U_{L-B}$ and $\Delta U_{B-L}$. (b) Energy $E_{M}$ (in $10^{-3} \mathrm{~nJ}$ ) of the local maximum as a function of $\epsilon$; see Eq. (5). The blue dots display the reconstruction from the measurement of the energetic barrier $\Delta U_{L-B}$ and the energy of a line, and the red squares represent the reconstruction from the measurement of the energetic barrier $\Delta U_{B-L}$ and the energy of a bubble. The origin of the energy is the line configuration energy, $E_{L}$. 
Moreover, the study of the mean residence times in each configuration, measured in this intermittent regime, gives a unique opportunity to precisely determine the barrier height of the energy landscape of the considered system. Lastly, from these values obtained at high temperature, we are able to determine the extension of the hysteresis loop that would exist at $T=0 \mathrm{~K}$

\section{APPENDIX A: NUMERICS}

The details on the numerical algorithm may be found in Ref. [37]. A short summary is presented here. We simulate identical point particles of mass $m$ moving on a plane, submitted to a thermal bath at temperature $T$. The thermal bath is accounted for by a damping constant $\gamma$ and by random forces applied on each particle, with the statistical properties of uncorrelated white Gaussian noise. The particles are transversally confined in a quasi-1D geometry by a harmonic potential of stiffness $\beta$, whereas periodic boundary conditions are applied in the longitudinal direction. The particles interact with a repulsive potential, which in all simulations is a screened electrostatic interaction $U(r)=U_{0} K_{0}\left(r / \lambda_{0}\right)$ with energy scale $U_{0}$, with a characteristic length $\lambda_{0}$ and where $K_{0}$ is the modified Bessel function of index 0 . The dynamics of the system is then simulated by the numerical integration of coupled Langevin equations [37].

Let $L$ be the length of the periodic simulation cell, and let $N$ be the number of particles. For all the simulations considered here, we have taken $N=128$ and $L=240 \mathrm{~mm}$, thus an interparticle distance $d=1.875 \mathrm{~mm}$. The damping constant is $\gamma=1 \mathrm{~s}^{-1}$. For such parameters, the equilibrium properties of the system have been described by a subcritical pitchfork bifurcation (see Appendix B and Ref. [24]).

The focus in this paper is on the influence of temperature on subcritical bifurcation. Thus the range of temperature considered here is between $10^{9}$ and $2 \times 10^{11} \mathrm{~K}$, to be compared with the typical interaction energy between particles at our concerned density $U_{\text {inter }} \approx 0.117 \mathrm{~nJ}\left(U_{\text {inter }} \approx 8.46 \times 10^{12} \mathrm{~K}\right)$.

In some simulations, we vary the stiffness by steps. The typical duration of a stiffness step is $2 \times 10^{5}$ time steps, and with a relative stiffness variation of $10^{-3}$. To get enough statistics for the PDF of $h_{m}(\epsilon, T, t)$, we have also undertaken longer simulations (up to $10^{7}$ time steps) at fixed confinement. Therefore, the analysis of the residence times has been obtained by the measure of at least 1000 jumps between each configuration for every confinement studied. The smoothness of the resulting distributions (see Fig. 9) attests to the quality of these measurements.

\section{APPENDIX B: BIFURCATION ANALYSIS OF THE ZIGZAG TRANSITION}

The normal form for the zigzag transition has been established in Ref. [24]. In this Appendix, we summarize the main results.

\section{Thermodynamic limit}

The zigzag transition happens because it is energetically favorable for the particles to organize in a staggered row when the density $1 / d$ increases or when the transverse stiffness $\beta$ decreases. In this zigzag pattern, the particles alternate with a transverse coordinate $\pm h$ so that the nearest-neighbor distance increases. Therefore, a decrease in the interaction energy eventually compensates for the energy increase due to the transverse confining potential. Since the two symmetric zigzag patterns have the same energy, the zigzag transition is a pitchfork bifurcation that takes place at a critical value $\beta_{Z Z}$ of the transverse stiffness. The physical origin of the normal form for this bifurcation is the coupling between the soft mode at the transition and the Goldstone mode due to the translational invariance of the infinite system.

A consistent expansion up to the power $\epsilon^{5 / 3}$, where $\epsilon$ is the distance to threshold $\beta=\beta_{Z Z}(1-\epsilon)$, gives the normal form as

$$
m \ddot{h}=\beta_{Z Z} \epsilon h+g_{3} h^{3}-g_{5} h^{5}+g_{1} \partial^{2} h / \partial x^{2},
$$

where all distances are in units of $d$ and where $\dot{h}$ denotes the time derivative of $h$. Knowing the interaction potential $U\left(r / \lambda_{0}\right)$ (see Appendix A), and restricting the interactions up to second neighbors only, we have given in Ref. [24] the analytic expressions of $\beta_{Z Z}$ and the coefficients $g_{i}$ that are known functions of $d$. For $d / \lambda_{0}>2.04$ (see [24]), the coefficients $g_{3}$ and $g_{5}$ in Eq. (B1) are positive and the pitchfork bifurcation is subcritical.

The line $h=0$ is a stable equilibrium pattern when $\epsilon \leqslant 0$. The homogeneous zigzag pattern is stable when $-g_{3}^{2} /\left(4 \beta_{Z Z} g_{5}\right) \leqslant \epsilon$, and its amplitude is

$$
h_{Z Z}^{2}=\frac{1}{2 g_{5}}\left(g_{3}+\sqrt{g_{3}^{2}+4 \epsilon \beta_{Z Z} g_{5}}\right) .
$$

In Ref. [24], we have shown that the normal form Eq. (B1) allows inhomogeneous stationary solutions when $-3 g_{3}^{2} /\left(16 \beta_{Z Z} g_{5}\right) \leqslant \epsilon \leqslant 0$, such that

$$
h(x)=\frac{h_{b-} h_{b+}}{\sqrt{\left(h_{b+}^{2}-h_{b-}^{2}\right) \cosh ^{2}\left(\sqrt{\frac{-\epsilon \beta_{Z Z}}{g_{1}}} x\right)+h_{b-}^{2}}},
$$

where

$$
h_{b \pm}^{2}=\frac{3}{2 g_{5}}\left(\frac{g_{3}}{2} \pm \sqrt{\frac{g_{3}^{2}}{4}+\frac{4}{3} \epsilon g_{5} \beta_{Z Z}}\right) .
$$

We call these excitations bubbles. In the context of phase transitions, these localized excitations exhibit a phase coexistence between the two phases (aligned and zigzag), hence a first-order transition. Their observation in our simulations evidences the subcriticality of the zigzag bifurcation.

\section{Periodic boundary conditions}

The previous analysis may be extended to finite systems with periodic boundary conditions, because the periodicity implies a rotational invariance that induces a Goldstone mode. The normal form (B1) is thus relevant to periodic systems, assuming, as for systems in the thermodynamic limit, the vicinity of the bifurcation threshold $(|\epsilon| \ll 1)$ and slow spatial variations $(|\partial h / \partial x| \ll h / d)$.

Periodic boundary conditions are relevant for the simulations, which exhibit localized inhomogeneous patterns, as shown in Ref. [24] and in Sec. II. In Ref. [24], the simulations 

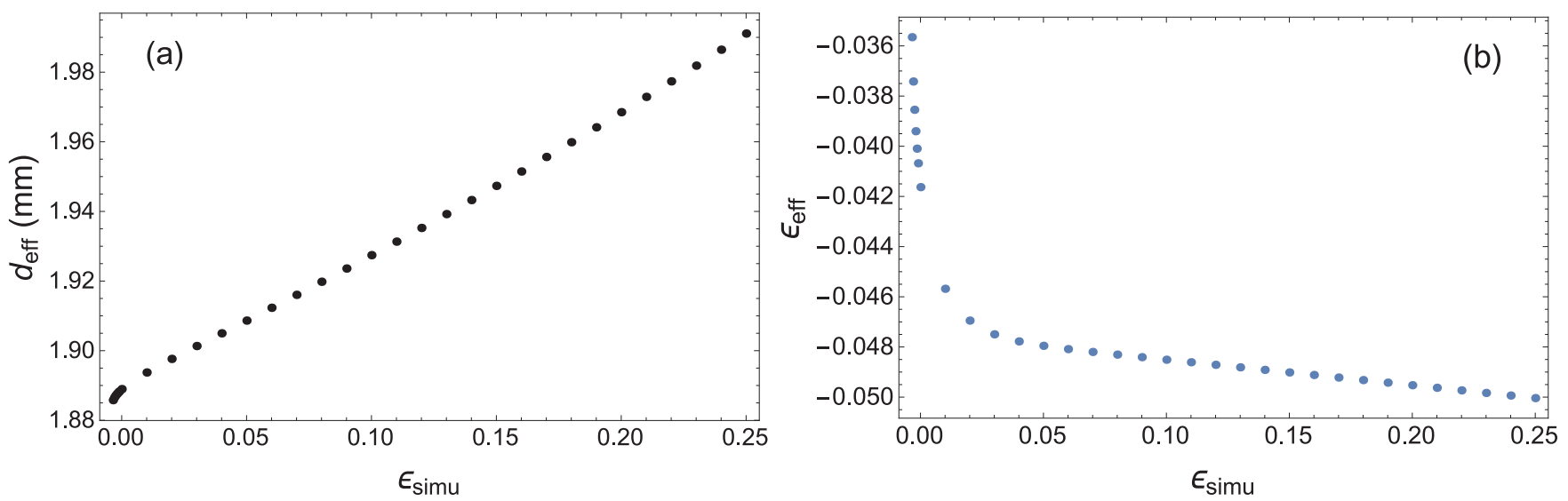

FIG. 13. (a) Plot of the numerical solution $d_{\text {eff }}(\mathrm{mm})$ of (B6) as a function of $\epsilon_{\text {simu }}$ for $N=128$ and $L=240 \mathrm{~mm}$. (b) Plot of the effective distance to threshold $\epsilon_{\text {eff }}$ as a function of $\epsilon_{\text {simu }}$ for $N=128$ and $L=240 \mathrm{~mm}$.

are done at very low temperature. The thermal fluctuations are very small, so that it is easy to measure the interparticle distance in that part of the inhomogeneous pattern where the particles are aligned (outside the bubble), which is found to be $d_{\text {eff }}>d$. It is therefore energetically favorable to have a localized excitation with nonzero transverse displacement $h(x)$, because the particles that stay aligned with $h=0$ are separated by a distance $d_{\text {eff }}$, which decreases their interaction energy.

In the simulations, the inhomogeneous patterns are observed when the stiffness $\beta_{\text {simu }}$ of the confining potential is such that $\beta_{\text {simu }}<\beta_{Z Z}(d)$. This defines a positive parameter $\epsilon_{\text {simu }}$ as $\beta_{\text {simu }}=\beta_{Z Z}(d)\left(1-\epsilon_{\text {simu }}\right)$. From the interparticle distance $d_{\text {eff }}>d$, we define an effective bifurcation parameter $\epsilon_{\mathrm{eff}}$,

$$
\epsilon_{\mathrm{eff}}=1-\frac{\beta_{\text {simu }}}{\beta_{Z Z}\left(d_{\mathrm{eff}}\right)} .
$$

We have shown in Ref. [24] that a bubble observed in a finite periodic system is very well described as a bubble in an infinite system, with an interparticle distance $d_{\text {eff }}$ and a distance to threshold $\epsilon_{\text {eff }}$.

At high temperature, it is less easy to measure $d_{\text {eff }}$ because of the thermal fluctuations. The coupling between the two soft modes, which is the basic mechanism responsible for the subcriticality of the zigzag transition, and thus for the possibility to observe the zigzag bubbles, leads to an analytic expression for the length of a periodic system of $N$ particles that includes a bubble. This length has to be equal to the spatial period $L$, which is expressed by [24]

$$
L=d_{\mathrm{eff}}\left[N-\frac{2 \alpha h_{b-} h_{b+}}{\kappa^{\prime}} \operatorname{arctanh}\left(\frac{h_{b-}}{h_{b+}}\right)\right]
$$

Here $\alpha$ is a known function of $d_{\text {eff }}$ that quantifies the mode coupling, and for a given value of the transverse stiffness $\beta_{\text {simu }}$ this is an equation of the only unknown $d_{\text {eff }}$.

The numerical roots $d_{\text {eff }}(\mathrm{mm})$ of (B6) and the corresponding effective distance to threshold $\epsilon_{\text {eff }}$ are plotted in Fig. 13 for several values of $\epsilon_{\text {simu }}$ [38]. We see that the calculated values of $d_{\text {eff }}$ are always greater than $d$, and that $\epsilon_{\text {eff }}$ is negative, which is consistent with our description of the bubbles.

In Fig. 2 we compare the bubble patterns observed at various temperatures with the analytic expression (B3), calculated for the value of $d_{\text {eff }}$ that is the root of (B6) for the value $\epsilon_{\text {simu }}$ and the particle number $N$ used in the simulations. We see that when the temperature is low enough to avoid the pattern destruction by the thermal fluctuations, the agreement is excellent.
[1] I. Waki, S. Kassner, G. Birkl, and H. Walther, Observation of Ordered Structures of Laser-Cooled Ions in a Quadrupole Storage Ring, Phys. Rev. Lett. 68, 2007 (1992).

[2] D. H. E. Dubin, Theory of Structural Phase Transitions in a Trapped Coulomb Crystal, Phys. Rev. Lett. 71, 2753 (1993).

[3] J. P. Schiffer, Phase Transitions in Anisotropically Confined Ionic Crystals, Phys. Rev. Lett. 70, 818 (1993).

[4] D. G. Enzer, M. M. Schauer, J. J. Gomez, M. S. Gulley, M. H. Holzscheiter, P. G. Kwiat, S. K. Lamoreaux, C. G. Peterson, V. D. Sandberg, D. Tupa, A. G. White, R. J. Hughes, and D. F. V. James, Observation of Power-Law Scaling for Phase Transitions in Linear Trapped Ion Crystals, Phys. Rev. Lett. 85, 2466 (2000).

[5] S. Seidelin, J. Chiaverini, R. Reichle, J. J. Bollinger, D. Leibfried, J. Britton, J. H. Wesenberg, R. B. Blakestad, R.
J. Epstein, D. B. Hume, W. M. Itano, J. D. Jost, C. Langer, R. Ozeri, N. Shiga, and D. J. Wineland, Microfabricated Surface-Electrode Ion Trap for Scalable Quantum Information Processing, Phys. Rev. Lett. 96, 253003 (2006).

[6] J. P. Home, D. Hanneke, J. D. Jost, D. Leibfried, and D. J. Wineland, Normal modes of trapped ions in the presence of anharmonic trap potentials, New J. Phys. 13, 073026 (2011).

[7] H. Landa, B. Reznik, J. Brox, M. Mielenz, and T. Schaetz, Structure, dynamics and bifurcations of discrete solitons in trapped ion crystals, New J. Phys. 15, 093003 (2013).

[8] M. Mielenz, J. Brox, S. Kahra, G. Leschhorn, M. Albert, T. Schaetz, H. Landa, and B. Reznik, Trapping of TopologicalStructural Defects in Coulomb Crystals, Phys. Rev. Lett. 110, 133004 (2013). 
[9] H. L. Partner, R. Nigmatulin, T. Burgermeister, K. Pyka, J. Keller, A. Retzker, M. B. Plenio, and T. E. Mehlstaubler, Dynamics of topological defects in ion Coulomb crystals, New J. Phys. 15, 103013 (2013).

[10] K. Pyka, J. Keller, H. L. Partner, R. Nigmatulin, T. Burgermeister, D. M. Meier, K. Kuhlman, A. Retzker, M. B. Plenio, W. H. Zurek, A. del Campo, and T. E. Mehlstaubler, Topological defect formation and spontaneous symmetry breaking in ion Coulomb crystals, Nat. Commun. 4, 2291 (2013).

[11] S. Ulm, J. Rossnagel, G. Jacob, C. Degunther, S. T. Dawkins, U. G. Poschinger, R. Nigmatulin, A. Retzker, M. B. Plenio, F. Schmidt-Kaler, and K. Singer, Observation of the Kibble-Zurek scaling law for defect formation in ion crystals, Nat. Commun. 4, 2290 (2013).

[12] H. Landa, A. Retzker, T. Schaetz, and B. Reznik, Entanglement Generation Using Discrete Solitons in Coulomb Crystals, Phys. Rev. Lett. 113, 053001 (2014).

[13] H. L. Partner, R. Nigmatulin, T. Burgermeister, J. Keller, K. Pyka, M. B. Plenio, A. Retzker, W. H. Zurek, A. del Campo, and T. E. Mehlstaubler, Structural phase transitions and topological defects in ion Coulomb crystals, Physica B 460, 114 (2015).

[14] T. Dessup, T. Maimbourg, C. Coste, and M. Saint Jean, Linear instability of a zigzag pattern, Phys. Rev. E 91, 022908 (2015).

[15] G. E. Astrakharchik, G. De Chiara, G. Morigi, and J. Boronat, Thermal and quantum fluctuations in chains of ultracold polar molecules, J. Phys. B 42, 154026 (2009).

[16] A. V. Straube, R. P. A. Dullens, L. Schimansky-Geier, and A. A. Louis, Zigzag transitions and nonequilibrium pattern formation in colloidal chains, J. Chem. Phys. 139, 134908 (2013).

[17] B. Liu and J. Goree, Phonons in a one-dimensional Yukawa chain: Dusty plasma experiment and model, Phys. Rev. E 71, 046410 (2005).

[18] A. Melzer, Zigzag transition of finite dust clusters, Phys. Rev. E 73, 056404 (2006).

[19] T. E. Sheridan, Dusty plasma ring model, Phys. Scr. 80, 065502 (2009).

[20] T. E. Sheridan and K. D. Wells, Dimensional phase transition in small Yukawa clusters, Phys. Rev. E 81, 016404 (2010).

[21] T. E. Sheridan and A. L. Magyar, Power law behavior for the zigzag transition in a Yukawa cluster, Phys. Plasmas 17, 113703 (2010).

[22] J.-B. Delfau, C. Coste, and M. Saint Jean, Transverse singlefile-diffusion near the zigzag transition, Phys. Rev. E 87, 032163 (2013).

[23] J.-B. Delfau, C. Coste, and M. Saint Jean, Noisy zigzag transition, fluctuations, and thermal bifurcation threshold, Phys. Rev. E 87, 062135 (2013).

[24] T. Dessup, C. Coste, and M. Saint Jean, Subcriticality of the zigzag transition: A nonlinear bifurcation analysis, Phys. Rev. E 91, 032917 (2015).
[25] The transition also happens when the density is increased, but it is by far a less suitable control parameter. The stiffness $\beta$ will be our control parameter henceforward, and the average density is a constant.

[26] G. Piacente, G. Q. Hai, and F. M. Peeters, Continuous structural transitions in quasi-one-dimensional classical Wigner crystals, Phys. Rev. B 81, 024108 (2010).

[27] J.-B. Delfau, Diffusion et corrélations de particules confinées en interaction à longue portée (in French), Ph.D. thesis, Université Paris Diderot, 2013.

[28] D. Forster, Hydrodynamic Fluctuations, Broken Symmetry, and Correlation Functions (Addison Wesley, New York, 1990).

[29] T. Dessup, C. Coste, and M. Saint Jean (unpublished).

[30] G. Agez, C. Szwaj, E. Louvergneaux, and P. Glorieux, Noisy precursors in one-dimensional patterns, Phys. Rev. A 66, 063805 (2002).

[31] G. Agez, M. G. Clerc, and E. Louvergneaux, Universal shape law of stochastic supercritical bifurcations: Theory and experiments, Phys. Rev. E 77, 026218 (2008).

[32] G. Agez, M. G. Clerc, E. Louvergneaux, and R. G. Rojas, Bifurcations of emerging patterns in the presence of additive noise, Phys. Rev. E 87, 042919 (2013).

[33] N. G. van Kampen, Stochastic Processes in Physics and Chemistry (North-Holland, Amsterdam, 1992).

[34] Note that for a given temperature and confinement these mean residence times are by definition such that $\left\langle\tau_{L}\right\rangle=\tau_{L-B}$ and $\left\langle\tau_{B}\right\rangle=\tau_{B-L}$.

[35] H. A. Kramers, Brownian motion in a field of force and the diffusion model of chemical reactions, Physica 7, 284 (1940).

[36] Notice that this procedure is well adapted when the temperature is high enough to allow jumps between the two local minima. Otherwise, noise-enhanced stability may happen and a nonmonotonic behavior of the residence time as a function of the additive thermal noise may be observed [39-41].

[37] J.-B. Delfau, C. Coste, and M. Saint Jean, Single file diffusion of particles with long-ranged interactions: Damping and finite size effects, Phys. Rev. E 84, 011101 (2011).

[38] To establish Eq. (B6), we have to calculate an integral that extends at infinity (see [24]). For this calculation to make sense for a finite periodic cell, the width of the bubble for which $h(x)$ is significantly different from 0 has to be small in comparison to the cell length $L$. The limiting upper value of $\epsilon_{\text {simu }}$ in Fig. 13 ensures that the bubble width is less than $L / 2$.

[39] N. V. Agudov and B. Spagnolo, Noise-enhanced stability of periodically driven metastable states, Phys. Rev. E 64, 035102 (2001)

[40] B. Spagnolo, N. V. Agudov, and A. A. Dubkov, Noise enhanced stability, Acta Phys. Pol. B 35, 1419 (2004).

[41] A. Fiasconaro, B. Spagnolo, and S. Boccaletti, Signatures of noise-enhanced stability in metastable states, Phys. Rev. E 72, 061110 (2005). 